\title{
Postoperative Pancreatic Fistula Following Traumatic Splenectomy: What Techniques Affect Incidence?
}

Peter Arnold ${ }^{1}$, Ashley Meagher ${ }^{1,2}$, Jennifer Hartwell ${ }^{1,2}$

${ }^{1}$ Indiana University School of Medicine, Indianapolis, IN, USA; ${ }^{2}$ Indiana University Health Methodist Hospital, Acute Care Surgery Services, Indianapolis, IN, USA

\section{Background and Hypothesis:}

Development of postoperative pancreatic fistula (POPF) in cases of adult splenectomy following trauma occur in 1-3\% of cases. Incidence of this complication leads to increased mortality rates, length-of-stays (LOS), and costs for patients. In cases where no previous pancreatic injury was noted, we hypothesized that the use of sutures in splenic hilum ligation instead of staples reduced the rate of incidence.

\section{Methods:}

Adult trauma patients (age $\geq 17$ years) that underwent non-elective splenectomy from 2010-2020 at Indiana University Methodist Hospital were retrospectively evaluated from the hospital's trauma registry. Due to the low incidence of POPF, our review was not adequately powered to perform statistical analysis with integrity; thus, we report our findings as a case series.

\section{Results:}

262 adult splenectomies following trauma occurred between 2010 and 2020; 160 passed exclusion criteria and were included in our analysis. Sutures alone across hilar structures were used in 135 cases, 6 of which developed POPF (4.4\%). Staples alone across hilar structures were used in 13 cases, 3 of which developed POPF (23\%). A combination of staples and sutures was used in 10 cases, none of which developed POPF. In cases of POPF, individuals averaged hospital LOS of 31.6 days, ICU LOS of 15.8 days, and 47.3 days with an abdominal drain in place. These patients averaged 4.78 abdominal CT scans, 1.11 endoscopic retrograde cholangio-pancreatography (ERCP), and 0.78 drain exchanges performed.

\section{Conclusion:}

Though not statistically significant, we noted that POPF are more likely to develop when staples alone are used across hilar structures. In our 10-year retrospective review of POPF, we found the complication to be rare but morbid, with associated prolonged length of hospital and ICU stays and requiring multiple imaging studies and interventions, leading to increased costs. We conclude that further study of optimal technique for emergent splenectomy is warranted. 